\title{
Anders gekommen
}

Liebe Leserinnen, liebe Leser,

als Jesus hörte, dass von inm gesagt werde, er treibe den Teufel mit Beelzebub aus, wies er das zurück und bemerkte dazu: Es könne nicht sein, denn das Böse hätte keinen Bestand, wenn es sich nicht einig wäre.,Ein jeglich Reich, so es mit sich selbst uneins wird, das wird wüst ...'1 Daran mochten viele gedacht haben, als die Rechtseinrichtung des Datenschutzbeauftragten vorgeschlagen wurde. Die Unternehmensleitung sollte vom Gesetz in die Pflicht genommen werden und der Datenschutzbeauftragte, ein ihr zu Treue verpflichteter Arbeitnehmer, sollte sicher stellen, dass sie der Pflicht nachkommt. Das könne doch nicht angehen, sagten die Zweifler. Zur Kontrolle brauche der Datenschutzbeauftragte Biss. Man könne aber nicht erwarten, dass er in die Hand beißt, die inn füttert. Diese Ansicht wurde noch ausgesprochener als bei uns im Ausland vertreten. Dort amüsierte man sich stellenweise über die Deutschen, die sich hier wie so oft mit Eifer über einfache Logik hinwegsetzen wollten. Die Einsetzung eines von der Unternehmensleitung abhängigen Datenschutzbeauftragten, der sie kontrollierten sollte, also eine Selbstkontrolle des Unternehmens, sei widersprüchlich, unzumutbar und unpraktikabel.

Das war nicht einfach von der Hand zu weisen. Sollte man aber deshalb eine zusätzliche unabhängige Gewalt schaffen? Das, fand man, ginge zu weit, zumindest soweit es die öffentliche Verwaltung betreffe. Sie sei institutionell zu Wohlverhalten verpflichtet. Deshalb könnten die öffentlichen Datenschutzbeauftragten trotz ihrer Kontrollfunktion dazu gehören. Der privaten, dem freien Markt dienenden Wirtschaft hingegen traute man weniger. Doch wollte man nicht für sie eine Kontrollbehörde einrichten, die entweder kostspielig oder uneffektiv geraten müsste. Die Firmen könnten sich selbst kontrollieren und so die Kosten tragen. Dafür erhielten sie einen Vertrauensvorschuss und der Gesetzgeber begnügte sich damit, die Kontrolleure von relativ schwach ausgestatteten Aufsichtsbehörden kontrollieren zu lassen. Diese Regelung bekam der Datenschutzidee gut: Mit dem Bundesdatenschutzgesetz hatte man Tausende von Datenschutz-Protagonisten geschaffen, nahezu einen eigenen Berufsstand, ausreichend, um die Medien aufmerksam zu machen und den Datenschutzes jedermann nahe zu bringen.

Soweit gut für die Idee. Wie aber sollten die Unternehmen und ihre Datenschutzbeauftragten mit dem biblischen Widerspruch auskommen? Würde sich der von den Kritikern vorausgesagte Loyalitätskonflikt einstellen? Konnte er nicht durch die herkömmlichen strafgesetzlichen Anzeigepflichten und das BGB Maßregelungsverbot an Schärfe zugewinnen? Unnötige Sorgen. Es ist - wie so oft - anders gekommen, als gedacht. Und dabei lag es doch von Anfang an auf der Hand: Interne Konflikte sind nicht schlimm für das Unternehmen; sie sind betrieblicher Alltag; man hat gelernt, mit ihnen auszukommen; sie halten das Unternehmen lebendig. Konflikte mit dem Gesetz hingegen gehen an den Nerv des Unternehmens. Sie können fatal sein, gründet doch das Unternehmen als Rechtsperson auf dem Gesetz und muss es doch die Gesetze befolgen, wenn es Bestand haben will. So mochte wohl die private Wirtschaft während der Datenschutz-Gesetzesfindung eine ihr angenehmere Regelung befürwortet haben. Nun aber, da die umstrittene Regelung Gesetz war, wurde sie respektiert. Und der Datenschutzbeauftragte gefährdete nicht Unternehmensziele. Im Gegenteil, auch die Unternehmensleitung beauftragte ihn, dafür zu sorgen, dass das Gesetz im Unternehmen befolgt wird.

Also nicht der Konflikt im Unternehmen, liebe Leserinnen und Leser, und nicht das Loyalitätsproblem des Datenschutzbeauftragten waren die Gefahr, sondern zu vermeiden war der Konflikt mit dem Gesetz, damit das „Reich nicht wüst werde“.

Mit freundlichen Grüßen, Ihr

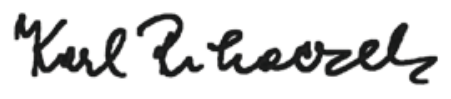

1 Lukas 11,15 